TITLE:

\title{
Molecular dynamics study of damage formation characteristics by large cluster ion impacts
}

$\operatorname{AUTHOR}(\mathrm{S}):$

Aoki, Takaaki; Matsuo, Jiro; Takaoka, Gikan

\section{CITATION:}

Aoki, Takaaki ... [et al]. Molecular dynamics study of damage formation characteristics by large cluster ion impacts. Nuclear Instruments and Methods in Physics Research Section B: Beam Interactions with Materials and Atoms 2003, 202: 278-282

\section{ISSUE DATE:}

2003-04

URL:

http://hdl.handle.net/2433/8945

\section{RIGHT:}

この論文は出版社版でありません。引用の際には出版社版をご確認ご 利用ください。; This is not the published version. Please cite only the published version. 
Molecular Dynamics Study of Damage Formation Characteristics by Large Cluster Ion Impacts Takaaki Aoki $^{1,2)}$, Jiro Matsuo ${ }^{1)}$ and Gikan Takaoka ${ }^{1)}$

1) Ion Beam Engineering Experimental Laboratory, Kyoto University

2) Collaborative Research Center of Cluster Ion Beam Process Technology

e-mail: t-aoki@kuee.Kyoto-u.ac.jp

Corresponding Author:

Takaaki Aoki

Ion Beam Engineering Experimental Laboratory, Kyoto University

Sakyo Kyoto, 606-8501, JAPAN

TEL: +81-75-753-4994 / FAX: +81-75-751-6774

e-mail: t-aoki@kuee.Kyoto-u.ac.jp

\section{$\underline{\text { Abstract }}$}

Molecular dynamics simulations of various Ar clusters, with the size ranging 1 to 50000 and the energy up to totally $50 \mathrm{keV}$, impacting on Si surfaces were performed. The cluster size and incident energy dependence on damage formation was examined. Two cluster-size depending parameters, the threshold energy to cause displacements and displacement yield, were proposed and modeled. These two parameters showed non-linearity depending on cluster size. The model function using above parameters reproduced the characteristics of damage formation by cluster ion impact. Two specific cluster sizes to cause maximum and no displacements on the surface were discussed, which is expected to provide useful information about optimized cluster ion beam conditions for various surface modification and thin film formation processes.

Keywords: Cluster impact, Molecular Dynamics, Damage Formation

PACS:

61.80.Lj (Atom and molecule irradiation effects)

36.40.-c (Atomic and molecular clusters)

45.50.-j (Dynamics and kinematics of a particle and a system of particles)

\section{$\underline{\text { INTRODUCTION }}$}

Cluster is an aggregation of large amount of molecules and its impact process on solid surface is very different from that of monomer ions. The difference is due to that, in the collisional process of cluster and surface, large number of interactions occur between cluster and surface atoms within very narrow region ( $\sim 100 \mathrm{~nm}$ square) and within very short time ( $\sim$ several ps). This high-density collisional process causes 'non-linear effect', which cannot be explained by the simple summation of independent event by monomer ion impact.

Various applications are expected by utilizing the non-linear effect of cluster ion impact [1-3]. The non-linear effect depends on various parameters of cluster ion irradiation, such like the atomic species, incident energy and cluster size. In order introduce the cluster ion beam process to industrial applications, it is important to clarify the relationship between the non-linear effect and the irradiation condition of cluster ion.

In this paper, the computer simulations of cluster ion impacting on solid surface were performed. The relationship between the number of displacements and energy and size of incident cluster were modeled. We discuss the suitable cluster size and energy for surface modification and thin-film formation.

\section{SIMULATION MODEL}

In order to study the collisional process between large cluster and solid surface, molecular 
dynamics (MD) simulations of Ar cluster impacting on $\mathrm{Si}(001)$ surface were performed. MD simulation is one method of computer simulation to solve numerically the Newton's equation of motion for each atom in the system using difference equation technique, so MD can make it possible to trace the time evolution of coordinates and velocity for every atom with high resolution. In this simulation, the interatomic potentials were described with Stillinger and Weber model [4] for Si-Si, and ZBL (Ziegler, Littmark, and Biersack) model [5] for Ar-Si, and Ar-Ar. The detail about simulation program is written in ref. [6].

Under recent experimental condition, large Ar gas cluster is generated by inletting high-pressure Ar gas source through laval nozzle [7]. The size of generated cluster ranges from several hundreds to several tens of thousands. Then, these neutral clusters are ionized, accelerated to several to several tens $\mathrm{keV}$, and irradiated on the solid target. In this work, the similar simulation condition to experiments were performed; Ar cluster with the size ranging from 1(monomer) to 50000 were irradiated at the energy of up to $50 \mathrm{keV}$. Large $\mathrm{Si}(001)$ target which consists of about 2000000 atoms, with the dimension of $400 \AA \times 400 \AA \times 200 \AA$ was prepared. This is because that irradiated Si target should be enough large to receive the energy induced by cluster impact; for example, when $20 \mathrm{keV}$ of total energy are deposited on the target, each target atom gains $0.025 \mathrm{eV}$.

In this study, the number of displacements at the cluster impact is examined. The displaced atom is defined as an atom which is below $2.5 \AA$ from the surface level and which potential energy is above $0.2 \mathrm{eV}$ from bulk status [8]. The MD simulations were performed for $8 \mathrm{ps}$, which is enough long to observe the impact process of cluster and solid target, such like penetration and re-evaporation of cluster atom, formation and relaxation of displaced target atoms.

\section{RESULTS AND DISCUSSION}

Fig. 1 shows the snapshots of $\mathrm{Ar}_{2000}$ clusters with various incident energies impacting on $\mathrm{Si}(001)$ surface, 8ps after the impact. Large and small circles indicate Ar and Si atom, respectively, and displaced $\mathrm{Si}$ atoms are indicated as small gray circiles. When incident energy is $10 \mathrm{eV} /$ atom (totally $20 \mathrm{keV}$ ), the Ar cluster penetrates the Si surface. Large number of Si atoms are displaced spherically and a crater-like damage remains on the surface after the re-evaporation of incident Ar atoms. This crater-like damage is confirmed by in-situ STM observation of Si surface bombarded with Ar cluster ions [9]. As the incident energy decreases, penetration depth of cluster, depth and radius of crater decreases. When the incident energy is as low as 1eV/atom, as shown in Fig. 1, the cluster does not penetrate the surface but break-up on the surface. During the collisional process, some surface atoms are displaced, but the displaced length is small, so that, these displacements recover and no damage remains on the surface within several pico-seconds after the impact.

The size and energy dependency of the number of displacements is examined using MD simulations. Fig. 2 shows the energy-per-atom $\left(E_{\mathrm{a}}\right)$ dependence of the number of displacements per atom $\left(D_{\mathrm{a}}\right)$ for various sizes of Ar clusters. $D_{\mathrm{a}}$ is given by dividing total number of displacements by the cluster size $N$. Fig. 2 indicates two $N$-depending trends;

1. There is a threshold incident energy $(T)$ to cause displacements in the target. $T$ depends on the cluster size.

2. If the incident energy is larger than $T, D_{\mathrm{a}}$ increases proportionally to the incident energy. The increasing ratio $(A)$ depends on the cluster size.

From these characteristics, the size and energy dependency of displacements can be modeled by the following formula;

$$
D_{\mathrm{a}}\left(N, E_{\mathrm{a}}\right)=A(N) \times\left(E_{\mathrm{a}}-T(N)\right),
$$

where $T$ and $A$ are aforementioned threshold energy and displacement formation yield, respectively. For each cluster size, fitting parameters for $T(N)$ and $A(N)$ in eq. (1) are calculated by least-square 
method. The size dependence of each parameter is shown in Fig. 3. As shown in Fig. 3, it is supposed that both $T(N)$ and $A(N)$ can be written in simple power-law equations;

$$
\begin{aligned}
& T(N)=T_{1} N^{\tau}, T_{1}=10.38[\mathrm{eV}], \tau=-0.25 \\
& A(N)=A_{1} N^{\alpha}, A_{1}=0.076[\text { atoms } / \mathrm{eV}], \alpha=0.26
\end{aligned}
$$

In eq. (2), $T_{1}$ and $A_{1}$ indicate the threshold energy and displacement formation yield for monomer ion, which are appropriate values compared to physical constant such like binding energy of Si-Si and parameters used by TRIM simulations. The power indices on $N, \tau$ and $\alpha$, mean the degree of non-linear effect. The decrease of threshold energy and the enhancement of displacement yield are due to high-density particle irradiation effect by cluster impact.

Eq. (1) and (2) lead the relationship between $N, E_{\mathrm{a}}$ and $D_{\mathrm{a}}$,

$$
D_{\mathrm{a}}\left(N, E_{\mathrm{a}}\right)=A(N) \times\left(E_{\mathrm{a}}-T(N)\right)=A_{1} N^{\alpha}\left(E_{\mathrm{a}}-T_{1} N^{\tau}\right),
$$

and the model function provides the total number of displacements $\left(D_{\mathrm{t}}\right)$,

$$
D_{\mathrm{t}}\left(N, E_{\mathrm{t}}\right)=N \times D_{\mathrm{a}}\left(N, E_{\mathrm{f}} / N\right)=A_{1}\left(N^{\alpha} E_{\mathrm{t}}-T_{1} N^{\alpha+\tau+1}\right),
$$

where $E_{\mathrm{t}}$ is total incident energy of the cluster and is equal to $N \times E_{\mathrm{a}}$. Fig. 4 shows the comparison of the model function and simulation results, size dependence of number total displacements by cluster impact. Two model functions at $E_{\mathrm{t}}=10 \mathrm{keV}, 20 \mathrm{keV}$ and $50 \mathrm{keV}$ are indicated by dashed lines, while symbols show the results calculated by MD simulations. The model function is formed from the results at $N<4000$, but it agrees with MD results at the region of $N>4000$ in the viewpoint that, at the $E_{\mathrm{t}}$ of $20 \mathrm{keV}$ for example, $D_{\mathrm{t}}$ reaches maximum at the cluster size of several thousands and gives 0 at the size of several tens of thousands. Eq. (4) gives the cluster sizes where a cluster causes no displacement $\left(N_{0}\right)$ or maximum number of displacements $\left(N_{\mathrm{m}}\right)$. These characteristic cluster sizes are given by,

$$
N_{0}=\left(E_{\mathrm{t}} / T_{1}\right)^{\frac{1}{\tau+1}}=\left(E_{\mathrm{t}} / T_{1}\right)^{1.33},
$$

and

$$
N_{\mathrm{m}}=\left(\frac{\alpha}{\alpha+\tau+1} \frac{E_{\mathrm{t}}}{T_{1}}\right)^{\frac{1}{\tau+1}}=0.146 N_{0} .
$$

Fig. 5 shows the $N_{0}$ and $N_{\mathrm{m}}$ for various total incident energy of cluster ion. Considering current irradiation condition of gas cluster ion beam ranges from several $\mathrm{keV}$ to several tens $\mathrm{keV}$ in acceleration energy, and from several hundreds to several tens of thousands in cluster size [6], it is supposed that various characteristic irradiation effect can be realized by selecting cluster size and incident energy. For example, when cluster ion is used for surface modification process, such as sputtering and surface smoothing, many surface atoms should be moved by cluster ion impact. Thus, it is considered that the preferable size distribution for these processes is around $N_{\text {m }}$; i.e. 4000 of mean size at $20 \mathrm{keV}$ of total incident energy. On the other hand, cluster ion assisted thin-film deposition is another expected application. In the case of thin film formation, it is important to deposit kinetic energy by ion impact without damage on the surface. As shown in Fig. 5, if the mean cluster size is 2000, required incident energy not to cause displacements on the surface is less than $3 \mathrm{keV}$. However, it is reported that high-quality thin film, such like diamond like carbon and transparent conductive film are formed at $5 \sim 7 \mathrm{keV}$ of incident energy [2,3]. It is considered that the energy deposition effect is not sufficient at 
the energy of $3 \mathrm{keV}$ and the size of 2000 , so the development of more large cluster formation is expected to establish more high-quality thin film formation technique using cluster ion beam.

\section{$\underline{\text { SUMMARY }}$}

Molecular dynamics simulations of large Ar cluster impacting on Si surface were performed. The cluster size and incident energy dependence on damage formation was examined. Two cluster-size depending parameters, the threshold energy to cause displacements and displacement yield, are introduced. These two parameters show non-linearity depending on cluster size. As the cluster size increases, the threshold energy decreases and the displacement yield increases. The model function using above parameters reproduced the characteristics of damage formation by cluster ion impact. When total incident energy is constant, the number of displacements increases with increasing cluster size, but there is specific number $\left(N_{\mathrm{m}}\right)$ to cause maximum number of displacements. If the cluster size is larger than that condition, the damage is reduced as the cluster size increases and reaches the cluster size where no displacement is generated $\left(N_{0}\right)$. These two specific cluster sizes, $N_{\mathrm{m}}$ and $N_{0}$, are expected to provide the information about suitable cluster ion irradiation conditions such like cluster size and irradiation energy for various surface modification and thin-film formation technologies.

\section{Acknowledgement}

The authors thankfully acknowledge NEDO (New energy and industrial technology development organization) in Japan for supporting this work.

\section{REFERENCES}

[1] I. Yamada, J. Matsuo, Z. Insepov, T. Aoki, T. Seki and N. Toyoda, Nucl. Instr. and Meth., B 164-165 (2000) 944.

[2] I. Yamada, T. Kitagawa, J. Matsuo and A. Kirkpatrick, Mass and charge transport in inorganic materials: fundamentals to devices, part B (Advances in science and technology 29), (2000) pp. 957,

[3] J. Matsuo, H. Katsumata, E. Minami and I. Yamada, Nucl. Instr. and Meth., B 161-163 (2000) 952.

[4] F. H. Stillinger and T. A. Weber, Phys. Rev., B 31 (1985) 5632.

[5] J. F. Ziegler, J. P. Biersack and U. Littmark, The stopping and range of ions in solids (Pergamon

Press, New York, U.S.A, 1985).

[6] T. Aoki, Molecular dynamics simulation of cluster ion impact on solid surface, Ph. D. thesis (Kyoto University, 2000)

[7] N. Toyoda, M. Saito, N. Hagiwara, J. Matsuo and I. Yamada, Proc. of 12th Ion Implantation Technology, (1998) 1234.

[8] M. J. Catula, T. diaz de la Rubia and G. H. Gilmer, Nucl. Instr. and Meth, B 196 (1995) 1.

[9] T. Seki, J. Matsuo, G. H. Takaoka and I. Yamada, Proc. of 16th international conference on the application of accelerators in research and industry, (AIP Conference Proceedings Vol. 576, 2001) 1003.

\section{FIGURE CAPTIONS}

Figure 1: Snapshots of $\mathrm{Ar}_{2000}$ cluster with various incident energy-per-atom impacting on $\mathrm{Si}(100)$ surface, 8ps after impact.

Figure 2: Incident energy dependence of the number of displacements for various sizes of Ar clusters. Figure 3: Cluster size dependence of two modeling parameters of damage formation by cluster impact - threshold energy to cause displacements (left) and displacement formation yield (right).

Figure 4: Cluster size dependence of total number of displacements by Ar clusters with total acceleration energies of 10, 20 and $50 \mathrm{keV}$. The dashed lines are model functions given by eq. (4).

Figure 5: Total energy dependence of the cluster size to no displacement (thick line) and maximum number of displacements (dashed line). 


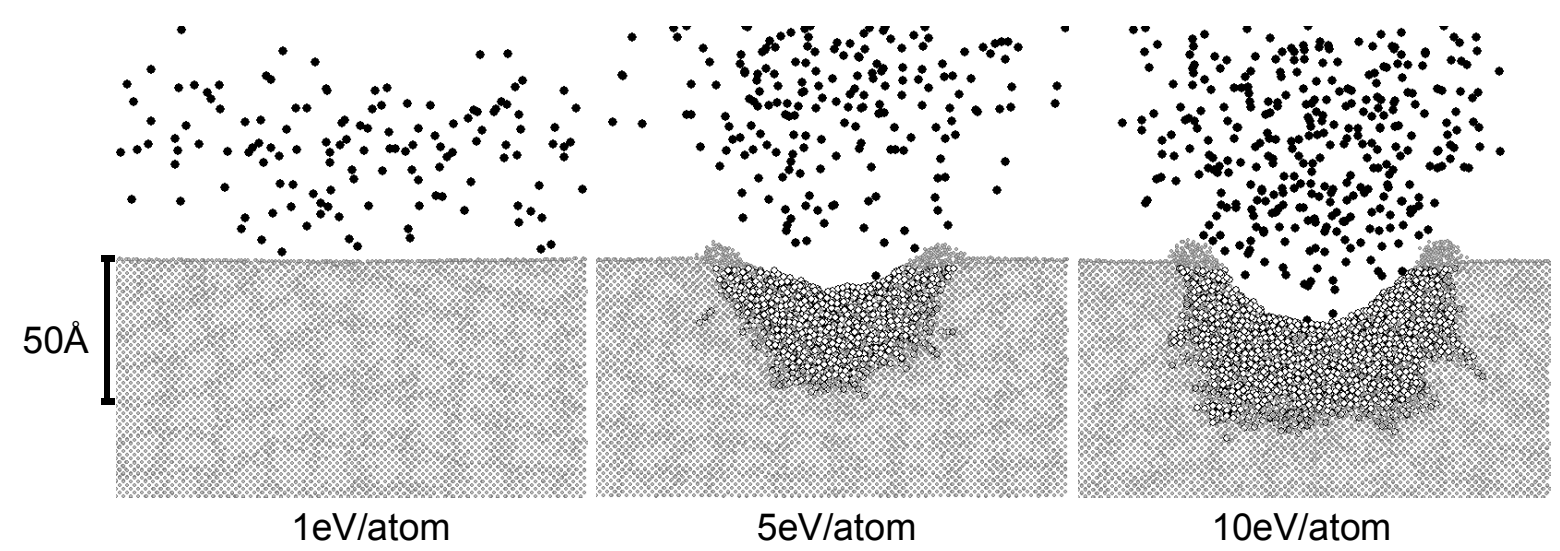

Figure 1: Snapshots of Ar2000 cluster with various incident energy-per-atom impacting on $\operatorname{Si}(100)$ surface, 8 ps after impact.

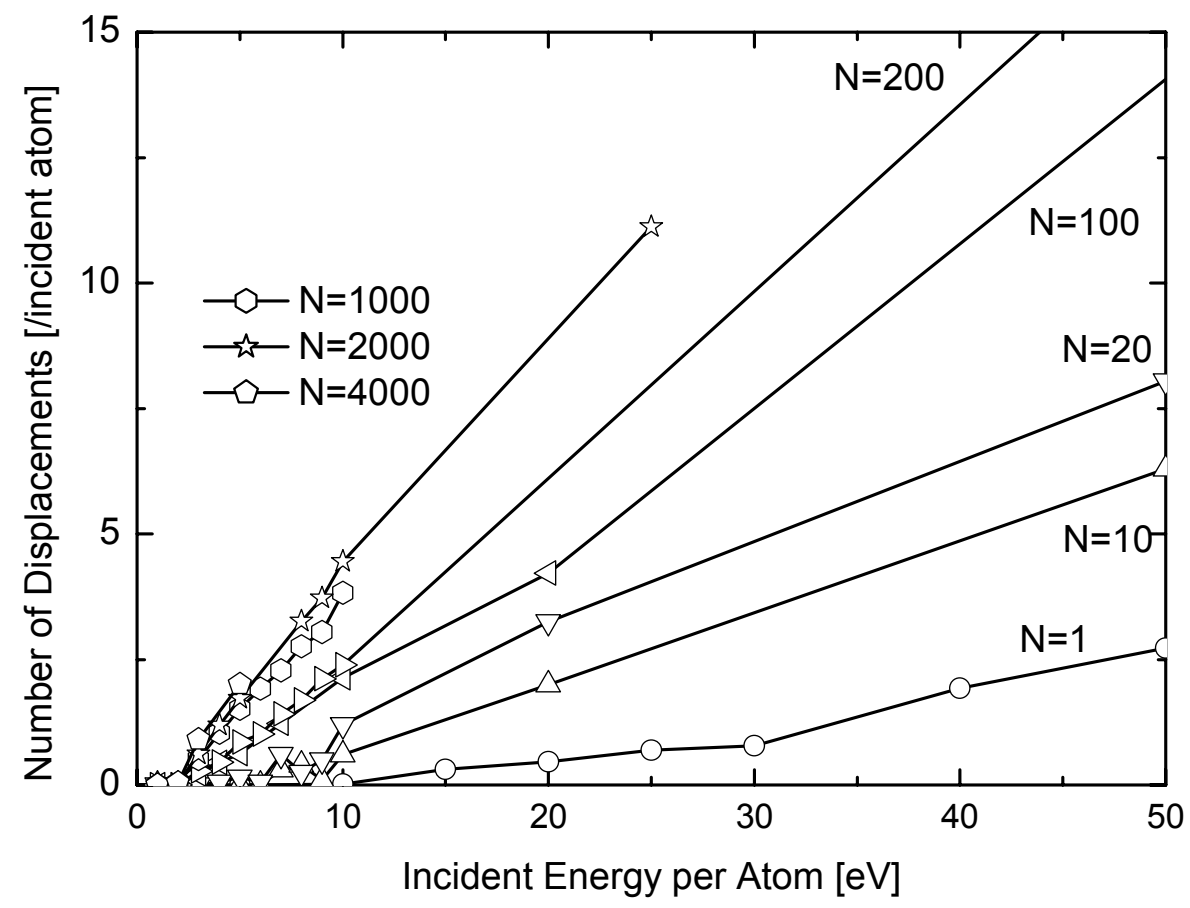

Figure 2: Incident energy dependence of the number of displacements for various sizes of Ar clusters. 

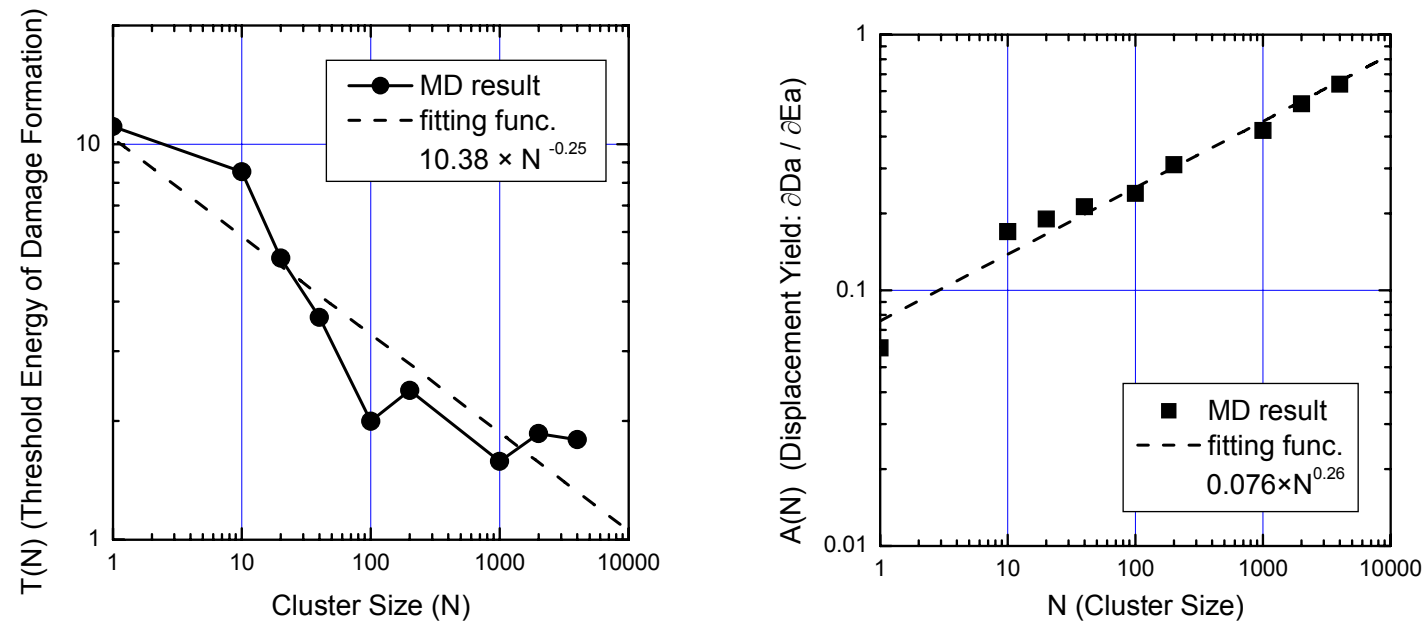

Figure 3: Cluster size dependence of two modeling parameters of damage formation by cluster impact threshold energy to cause displacements (left, $T$ ) and displacement formation yield (right, $A$ ).

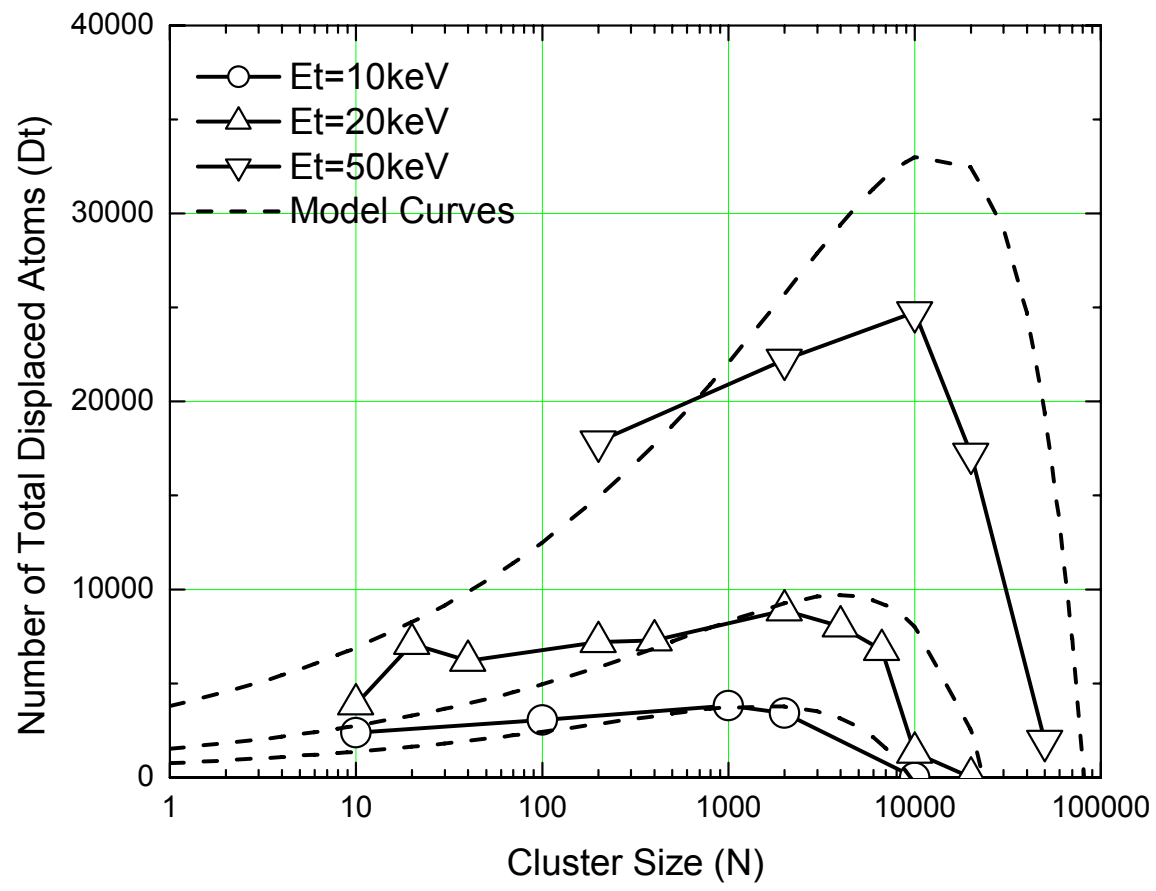

Figure 4: Cluster size dependence of total number of displacements by Ar clusters with total acceleration energies of 10, 20 and 50keV. The dashed lines are model functions given by eq. (4). 


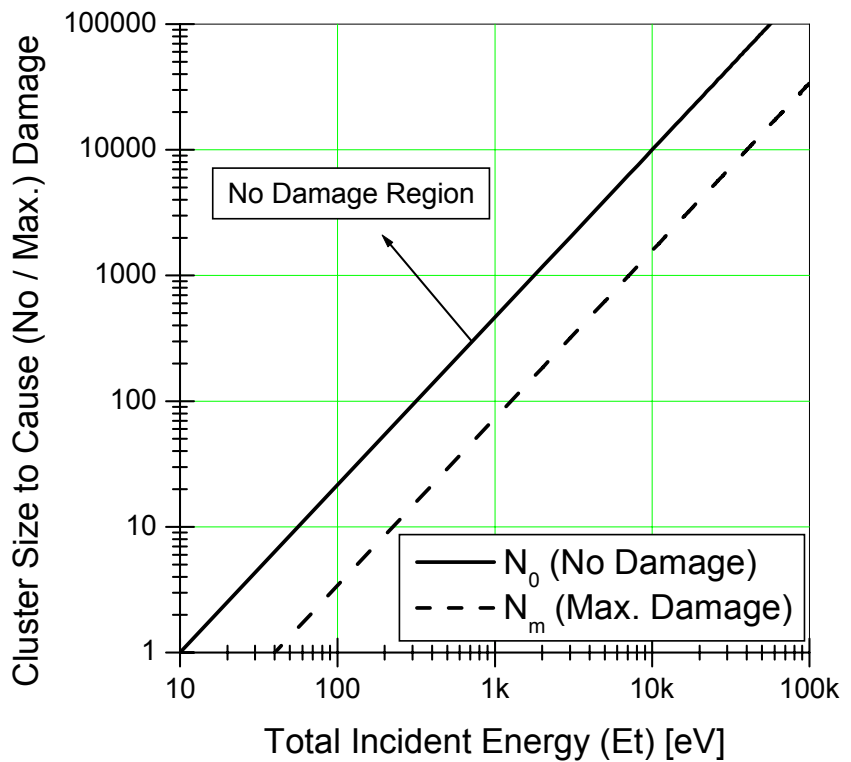

Figure 5: Total energy dependence of the cluster size to no displacement (thick line) and maximum number of displacements (dashed line). 